\title{
COMPLETE HYPERSURFACES WITH CONSTANT MEAN CURVATURE AND NON-NEGATIVE SECTIONAL CURVATURES
}

HU ZE-JUN

(Communicated by Christopher B. Croke)

\begin{abstract}
We classify the complete and non-negatively curved hypersurfaces of constant mean curvature in spaces of constant sectional curvature.
\end{abstract}

\section{INTRODUCTION}

Let $\bar{M}^{n+1}(c)$ be an $(n+1)$-dimensional space of constant sectional curvature $c$. When $c<0, \bar{M}^{n+1}(c)=H^{n+1}(c)$; when $c=0, \bar{M}^{n+1}(c)=R^{n+1}$; when $c>0, \bar{M}^{n+1}(c)=S^{n+1}(c)$, respectively. Let $M^{n}$ be an $n$-dimensional hypersurface with constant mean curvature $H$ in $\bar{M}^{n+1}(c)$. Let $S$ denote the square of the length of the second fundamental form. The main purpose of this paper is to give a characterization of non-negatively curved hypersurfaces of $\bar{M}^{n+1}(c)$ by the relationship between $S$ and $H$, this can be compared with the results obtained by Nomizu and Smyth [3], Okumura [4], Goldberg [1], Hasanis [2] and Smyth [6].

Theorem. Let $M^{n}$ be a complete non-negatively curved hypersurface of $\bar{M}^{n+1}(c)$ with constant mean curvature $H$. Then $M^{n}$ is totally umbilical or

$$
\sup S=n c+\frac{n^{3} H^{2}}{2 k(n-k)} \pm \frac{n(n-2 k)}{2 k(n-k)}|H| \sqrt{n^{2} H^{2}+4 k(n-k) c},
$$

for some $k=1,2, \ldots, n-1$, when $c \geq 0$; or

$$
\sup S=n c+\frac{n^{3} H^{2}}{2(n-1)}-\frac{n(n-2)}{2(n-1)}|H| \sqrt{n^{2} H^{2}+4(n-1) c}, \quad \text { when } c<0 .
$$

In particular, if $M^{n}$ is connected and $S=$ constant (when $c>0, S=$ constant may be replaced by the condition that $M^{n}$ is compact), then in the second case we have the following:

(1) When $c>0, M^{n}=S^{k}\left(c_{1}\right) \times S^{n-k}\left(c_{2}\right)$, for some $k=1,2, \ldots, n-1$, where $c_{1}>0, c_{2}>0$ and $1 / c_{1}+1 / c_{2}=1 / c$.

Received by the editors October 21, 1992 and, in revised form, February 3, 1994.

1991 Mathematics Subject Classification. Primary 53C40; Secondary 53C20.

Key words and phrases. Hypersurfaces of constant mean curvature, sectional curvature, totally umbilical. 
(2) When $c=0, M^{n}=R^{k} \times S^{n-k}\left(c_{1}\right)$ for some $k=1,2, \ldots, n-1$, where $c_{1}>0$.

(3) When $c<0, M^{n}=H^{1}\left(c_{1}\right) \times S^{n-1}\left(c_{2}\right)$, where $c_{1}<0, c_{2}>0$ and $1 / c_{1}+1 / c_{2}=1 / c$.

From the theorem, we easily see the following

Corollary. Let $M^{n}$ be a compact non-negatively curved hypersurface of $\bar{M}^{n+1}$ (c) $(c \leq 0)$ with constant mean curvature $H$. Then $M^{n}$ is totally umbilical and has positive sectional curvature $H^{2}+c$.

Remark. The main theorem was partially proved by Nomizu and Smyth in [3] whose results were extended to arbitrary codimension by Smyth [6] and later by Yau in [7] under the condition that $M^{n}$ is compact.

\section{Preliminaries}

Let $M^{n}$ be a hypersurface of $\bar{M}^{n+1}(c)$ and let $e_{1}, \ldots, e_{n}, e_{n+1}$ be a local field of orthonormal frames in $\bar{M}^{n+1}(c)$, such that, restricted to $M^{n}$, the vector field $e_{n+1}$ is normal to $M^{n}$. Then the second fundamental form $B$ and the mean curvature $H$ for $M^{n}$ can be written as

$$
B=\sum_{i, j} h_{i j} \omega_{i} \omega_{j} e_{n+1}, \quad H=(1 / n) \sum_{i} h_{i i} .
$$

The Gauss equation for $M^{n}$ is

$$
R=n(n-1) c+n^{2} H^{2}-S,
$$

where $S=\operatorname{tr} B^{2}=\sum_{i, j} h_{i j}^{2}$ and $R$ denotes the scalar curvature of $M^{n}$.

We denote by $\Delta$ the Laplacian relative to the induced metric on $M^{n}$. If $H=$ constant , then ([3])

$$
(1 / 2) \Delta S=|\nabla B|^{2}-S^{2}+n c S-n^{2} c H^{2}+n H \operatorname{tr} B^{3} .
$$

For any point $p$ in $M^{n}$, we can choose a local frame field $e_{1}, e_{2}, \ldots, e_{n}$ so that the matrix $\left(h_{i j}\right)$ is diagonalized at that point, say, $h_{i j}=\lambda_{i} \delta_{i j}$. Then $(2.3)$ can be rewritten as ([3])

$$
(1 / 2) \Delta S=|\nabla B|^{2}+\sum_{i<j}\left(\lambda_{i}-\lambda_{j}\right)^{2} K_{i j}
$$

where $K_{i j}=c+\lambda_{i} \lambda_{j}$ is the sectional curvature of the plane section spanned by $e_{i}$ and $e_{j}$.

Lemma (see Omori [5] and Yau [8]). Let $M^{n}$ be an $n$-dimensional complete Riemannian manifold whose Ricci curvature is bounded from below. Let $F$ be a $C^{2}$-function bounded from above on $M^{n}$. Then for any $\varepsilon>0$, there exists a point $p$ in $M^{n}$ such that

$$
\sup F-\varepsilon<F(p), \quad|\operatorname{grad} F|(p)<\varepsilon, \quad \Delta F(p)<\varepsilon .
$$

\section{STANDARD MODELS}

This section is concerned with some standard models of complete nonnegatively curved hypersurfaces with constant mean curvature of the space form 
$\bar{M}^{n+1}(c)$. In particular, we only consider non-totally umbilical cases, and the length of the second fundamental form of such hypersurfaces are calculated.

First, we consider a class of hypersurfaces $R^{k} \times S^{n-k}\left(c_{1}\right)$ of $R^{n+1}$, where $k=1,2, \ldots, n-1$. The number of distinct principal curvatures of each hypersurface is exactly two, say 0 and $\sqrt{c_{1}}$, with multiplicities $k$ and $n-$ $k$, respectively. The sectional curvatures of a plane spanned by two principal directions are 0 and $c_{1}$, respectively. It is easily seen that $H$ and $S$ are constant, and they satisfy, for $R^{k} \times S^{n-k}\left(c_{1}\right)$ in $R^{n+1}, S=n^{2} H^{2} /(n-k)$.

We next consider the case $c>0$. Let

$$
\begin{aligned}
S^{k}\left(c_{1}\right) & =\left\{\left(x_{1}, \ldots, x_{k+1}\right) \in R^{k+1} ; x_{1}^{2}+\cdots+x_{k+1}^{2}=1 / c_{1}\right\}, \\
S^{n-k}\left(c_{2}\right) & =\left\{\left(y_{1}, \ldots, y_{n-k+1}\right) \in R^{n-k+1} ; y_{1}^{2}+\cdots+y_{n-k+1}^{2}=1 / c_{2}\right\}, \\
S^{n+1}(c) & =\left\{\left(x_{1}, \ldots, x_{k+1}, y_{1}, \ldots, y_{n-k+1}\right) \in R^{n+2} ;\right. \\
& \left.x_{1}^{2}+\cdots+x_{k+1}^{2}+y_{1}^{2}+\cdots+y_{n-k+1}^{2}=1 / c\right\},
\end{aligned}
$$

where $1 / c_{1}+1 / c_{2}=1 / c, k=1,2, \ldots, n-1$. Then $S^{k}\left(c_{1}\right) \times S^{n-k}\left(c_{2}\right)$ is a family of hypersurfaces in $S^{n+1}(c)$. The number of distinct principal curvatures of such hypersurfaces in this family is exactly two, and they are constant. One principal curvature is equal to $\pm \sqrt{c_{1}-c}$ with multiplicity $k$, and the other is equal to $\mp \sqrt{c_{2}-c}$ with multiplicity $n-k$. The sectional curvatures of a plane spanned by two principal directions are $c_{1}, c_{2}$ and 0 , respectively. For $S^{k}\left(c_{1}\right) \times S^{n-k}\left(c_{2}\right)$ in $S^{n+1}(c)$ we can easily show that

$$
S=n c+\frac{n^{3} H^{2}}{2 k(n-k)} \pm \frac{n(n-2 k)}{2 k(n-k)}|H| \sqrt{n^{2} H^{2}+4 k(n-k) c},
$$

where the plus (resp. minus) sign is taken if $k \sqrt{c_{1}-c} \geq(n-k) \sqrt{c_{2}-c}$ (resp. $\left.k \sqrt{c_{1}-c}<(n-k) \sqrt{c_{2}-c}\right)$.

Finally, we consider the case $c<0$. Let

$$
H^{n+1}(c)=\left\{\left(x_{0}, x_{1}, \ldots, x_{n+1}\right) \in R_{1}^{n+2} ;-x_{0}^{2}+x_{1}^{2}+\cdots+x_{n+1}^{2}=1 / c\right\} .
$$

Define a family of hypersurfaces $H^{k}\left(c_{1}\right) \times S^{n-k}\left(c_{2}\right)$ in $H^{n+1}(c)$ by

$$
\begin{aligned}
H^{k}\left(c_{1}\right) \times S^{n-k}\left(c_{2}\right)= & \left\{\left(x_{0}, x_{1}, \ldots, x_{n+1}\right) \in R_{1}^{n+2} ;\right. \\
& \left.-x_{0}^{2}+x_{1}^{2}+\cdots+x_{k}^{2}=1 / c_{1}, x_{k+1}^{2}+\cdots+x_{n+1}^{2}=1 / c_{2}\right\},
\end{aligned}
$$

where $c_{1}<0, c_{2}>0,1 / c_{1}+1 / c_{2}=1 / c$ and $k=1,2, \ldots, n-1$. The number of distinct principal curvatures of such hypersurfaces in this family is exactly two and they are constant. One principal curvature is equal to $\sqrt{c_{1}-c}$ with multiplicity $k$ and the other is equal to $\sqrt{c_{2}-c}$ with multiplicity $n-k$. The sectional curvatures are $0, c_{2}$ and $c_{1}$ for $1<k<n$; they are 0 and $c_{2}$ for $k=1$. For $H^{k}\left(c_{1}\right) \times S^{n-k}\left(c_{2}\right)$ in $H^{n+1}(c)$, we can also easily show that

$$
S=n c+\frac{n^{3} H^{2}}{2 k(n-k)} \pm \frac{n(n-2 k)}{2 k(n-k)}|H| \sqrt{n^{2} H^{2}+4 k(n-k) c},
$$


where the plus (resp. minus) sign is taken if $k \sqrt{c_{1}-c} \geq(n-k) \sqrt{c_{2}-c}$ (resp. $\left.k \sqrt{c_{1}-c}<(n-k) \sqrt{c_{2}-c}\right)$.

\section{Proof of TheOREM}

Since $K_{i j}=c+\lambda_{i} \lambda_{j} \geq 0$ for any distinct indices $i$ and $j,(2.4)$ means that

$$
(1 / 2) \Delta S=|\nabla B|^{2}+\sum_{i<j}\left(\lambda_{i}-\lambda_{j}\right)^{2}\left(c+\lambda_{i} \lambda_{j}\right) \geq 0 .
$$

On the other hand, $K_{i j} \geq 0$ implies that the Ricci curvature of $M^{n}$ is bounded from below by zero. From (2.2) we have that $S$ is bounded from above by a constant $n(n-1) c+n^{2} H^{2}$. Then we can apply the lemma to the function $S$. Then we get $\left\{p_{m}\right\}$ in $M^{n}$ such that

$$
\lim _{m \rightarrow \infty} S\left(p_{m}\right)=\sup S, \quad \lim _{m \rightarrow \infty} \Delta S\left(p_{m}\right) \leq 0
$$

This implies that (4.1) and (4.2) give rise to

$$
\left(c+\lambda_{i} \lambda_{j}\right)\left(\lambda_{i}-\lambda_{j}\right)^{2}\left(p_{m}\right) \rightarrow 0 \quad(m \rightarrow \infty),
$$

for any distinct indices $i$ and $j$.

Now, since $S=\sum \lambda_{j}^{2}$ is bounded, any principal curvature $\lambda_{j}$ is bounded and hence so is any sequence $\left\{\lambda_{j}\left(p_{m}\right)\right\}$. Then there exists a subsequence $\left\{p_{m^{\prime}}\right\}$ of $\left\{p_{m}\right\}$ such that

$$
\lambda_{j}\left(p_{m^{\prime}}\right) \rightarrow \stackrel{\circ}{\lambda}_{j} \quad\left(m^{\prime} \rightarrow \infty\right) \text { for some } \stackrel{\circ}{\lambda}_{j} \text { and any } j .
$$

In fact, since a sequence $\left\{\lambda_{1}\left(p_{m}\right)\right\}$ is bounded, it converges to some $\stackrel{\circ}{\lambda}_{1}$ by taking a subsequence $\left\{p_{m_{1}}\right\}$ if necessary. For the point sequence $\left\{p_{m_{1}}\right\}$, a sequence $\left\{\lambda_{2}\left(p_{m_{1}}\right)\right\}$ is also bounded and hence there is a subsequence $\left\{p_{m_{2}}\right\}$ of $\left\{p_{m_{1}}\right\}$ such that $\left\{\lambda_{2}\left(p_{m_{2}}\right)\right\}$ converges to some $\lambda_{2}$ as $m_{2}$ tends to infinity. Thus we can inductively show that there exists a point sequence $\left\{p_{m^{\prime}}\right\}$ of $\left\{p_{m}\right\}$ such that the property (4.4) holds. By (4.3) and (4.4) we get

$$
\left(c+\stackrel{\circ}{\lambda}_{i} \stackrel{\circ}{j}_{j}\right)\left(\stackrel{\circ}{\lambda}_{i}-\stackrel{\circ}{\lambda}_{j}\right)^{2}=0,
$$

for any distinct indices $i$ and $j$. By a simple algebraic calculation it is easily seen that the number of distinct limits in $\left\{\hat{\lambda}_{i}\right\}$ is at most two

Case 1. If all limits $\dot{\lambda}_{i}$ coincide with each other, because $S-n H^{2}=\sum \lambda_{i}^{2}-$ $(1 / n)\left(\sum \lambda_{i}\right)^{2}=(1 / n) \sum_{i<j}\left(\lambda_{i}-\lambda_{j}\right)^{2}$, it follows from the above property that $\lim _{m_{2}^{\prime} \rightarrow \infty}\left(S-n H^{2}\right)\left(p_{m^{\prime}}\right)=0$. Combining this with (4.2), we get $\sup S=n H^{2}$. Hence the function $S$ becomes a constant $n H^{2}$. Accordingly, the hypersurface $M^{n}$ is totally umbilical.

Case 2. If $\left\{\dot{\lambda}_{i}\right\}$ has exactly two distinct elements, without loss of generality, we may set

$$
\stackrel{\circ}{\lambda}_{1}=\cdots=\stackrel{\circ}{\lambda}_{k}=\lambda, \quad \stackrel{\circ}{\lambda}_{k+1}=\cdots=\stackrel{\circ}{\lambda}_{n}=\mu, \quad \lambda<\mu,
$$

for some $k=1,2, \ldots, n-1$. From (4.5) we have

$$
\lambda \mu=-c \text {. }
$$


Now, we only consider the case $c<0$. As for the cases $c>0$ and $c=0$, the proof is simpler and similar to each other, we omit them.

Without loss of generality, we may assume that $H \geq 0$. Then two limits $\lambda$ and $\mu$ are positive. In this case we define a negative number $c_{1}$ and a positive number $c_{2}$ by $\lambda^{2}=c_{1}-c$ and $\mu^{2}=c_{2}-c$, respectively, so these numbers satisfy

$$
\left\{\begin{array}{l}
c<c_{1}<0, \quad c_{2}>0, \\
\sqrt{\left(c_{1}-c\right)\left(c_{2}-c\right)}=-c, \quad \text { i.e., } 1 / c_{1}+1 / c_{2}=1 / c .
\end{array}\right.
$$

By $H=(1 / n) \sum \lambda_{i}\left(p_{m^{\prime}}\right)=$ constant, we have

$$
n H=k \lambda+(n-k) \mu=k \sqrt{c_{1}-c}+(n-k) \sqrt{c_{2}-c} .
$$

At the same time we have

$$
\begin{aligned}
\sup S & =\lim _{m^{\prime} \rightarrow \infty} \sum \lambda_{i}^{2}\left(p_{m^{\prime}}\right)=k \lambda^{2}+(n-k) \mu^{2} \\
& =k\left(c_{1}-c\right)+(n-k)\left(c_{2}-c\right) .
\end{aligned}
$$

On the other hand, by using (4.7) and (4.8) we can easily see that

$$
\begin{aligned}
n c+ & \frac{n^{3} H^{2}}{2 k(n-k)} \pm \frac{n(n-2 k)}{2 k(n-k)}|H| \sqrt{n^{2} H^{2}+4 k(n-k) c} \\
& =k\left(c_{1}-c\right)+(n-k)\left(c_{2}-c\right),
\end{aligned}
$$

where the plus (resp. minus) sign is taken if $k \sqrt{c_{1}-c} \geq(n-k) \sqrt{c_{2}-c}$ (resp. $\left.k \sqrt{c_{1}-c}<(n-k) \sqrt{c_{2}-c}\right)$.

Thus, from (4.9) we have

$$
\sup S=n c+\frac{n^{3} H^{2}}{2 k(n-k)} \pm \frac{n(n-2 k)}{2 k(n-k)}|H| \sqrt{n^{2} H^{2}+4 k(n-k) c} .
$$

When $k>1$, the sectional curvature $K_{12}\left(p_{m^{\prime}}\right)=\left(c+\lambda_{1} \lambda_{2}\right)\left(p_{m^{\prime}}\right) \underset{m^{\prime} \rightarrow \infty}{\rightarrow} c+$ $\lambda^{2}=c_{1}<0$, so from the assumption we have $k=1$. Then $k \sqrt{c_{1}-c}<$ $(n-k) \sqrt{c_{2}-c}$. Therefore we have at last

$$
\sup S=n c+\frac{n^{3} H^{2}}{2(n-1)}-\frac{n(n-2)}{2(n-1)}|H| \sqrt{n^{2} H^{2}+4(n-1) c} .
$$

This completes the proof of the first part of the theorem.

In particular, if $S=$ constant and $M^{n}$ is connected, then (2.4) says that all the principal curvatures are constant and that they satisfy

$$
\left(c+\lambda_{i} \lambda_{j}\right)\left(\lambda_{i}-\lambda_{j}\right)^{2}=0,
$$

for any distinct indices $i$ and $j$. Hence the number of distinct elements in $\left\{\lambda_{i}\right\}$ is at most two.

If $\lambda_{1}=\cdots=\lambda_{n}$, then $M^{n}$ is totally umbilical.

If $\left\{\lambda_{i}\right\}$ has exactly two distinct elements, without loss of generality we may assume that $\lambda_{1}=\cdots=\lambda_{k}=\lambda, \lambda_{k+1}=\cdots=\lambda_{n}=\mu, \lambda<\mu$, for some $k=1,2, \ldots, n-1$. From (4.10) we have

$$
\lambda \mu=-c \text {. }
$$

For $c \geq 0$, the theorem has been proved by Nomizu and Smyth [3], so we just consider the case $c<0$. Without loss of generality we may assume that 
$H \geq 0$. Then, similar to the first part, we get $\lambda=\sqrt{c_{1}-c}, \mu=\sqrt{c_{2}-c}$ and $k=1$, where $c_{1}, c_{2}$ satisfy (4.7). Thus the second fundamental form of $M^{n}$ in $H^{n+1}(c)$ is given by

$$
\left(h_{i j}\right)=\operatorname{diag}\left(\sqrt{c_{1}-c}, \sqrt{c_{2}-c}, \ldots, \sqrt{c_{2}-c}\right) .
$$

Then, by using the method similar to that of [3] and combining with Section 3 , we can show that $M^{n}$ is isometric to $H^{1}\left(c_{1}\right) \times S^{n-1}\left(c_{2}\right)$. Q.E.D.

\section{ACKNOWLEDGMENTS}

I would like to express my deep gratitude to Professor Christopher B. Croke and the referee for their many helpful suggestions and their corrections on this paper.

\section{REFERENCES}

1. S. I. Goldberg, An application of Yau's maximum principle to conformally flat spaces, Proc. Amer. Math. Soc. 79 (1980), 268-270.

2. T. Hasanis, Characterization of totally umbilical hypersurfaces, Proc. Amer. Math. Soc. 81 (1981), 447-450.

3. K. Nomizu and B. Smyth, A formula of Simons' type and hypersurfaces with constant mean curvature, J. Differential Geom. 3 (1969), 367-377.

4. M. Okumura, Hypersurfaces and a pinching problem on the second fundamental tensor, Amer. J. Math. 96 (1974), 207-213.

5. H. Omori, Isometric immersions of Riemannian manifolds, J. Math. Soc. Japan 19 (1967), 205-214.

6. B. Smyth, Submanifolds of constant mean curvature, Math. Ann. 205 (1973), 265-280.

7. S. T. Yau, Submanifolds with constant mean curvature, Amer. J. Math. 97 (1975), 76-100.

8. Harmonic functions on complete Riemannian manifolds, Comm. Pure. Appl. Math. 28 (1975), 201-208.

Department of Mathematics, Zhengzhou University, Zhengzhou, 450052, Henan, PeoPLE'S REPUBLIC OF CHINA

Current address: Department of Mathematics, Sichuan University, Chengdu, 610064, Sichuan, People's Republic of China 